\title{
Laser patterning of amorphous silicon thin films deposited on flexible and rigid substrates
}

\author{
P. Alpuim ${ }^{1,2}$, M.F. Cerqueira1, V. Iglesias ${ }^{2}$, and J. Borme ${ }^{2}$ \\ ${ }^{1}$ Department of Physics, Universidade do Minho, Campus de Gualtar, 4715-057 Braga, Portugal \\ ${ }^{2}$ International Iberian Nanotechnology Laboratory, Av. Mestre José Veiga, 4715-330 Braga, Portugal
}

Received ZZZ, revised ZZZ, accepted ZZZ

Published online ZZZ (Dates will be provided by the publisher.)

Keywords Laser scribing; amorphous silicon; crystallization; dopant activation; piezoresistance.

\begin{abstract}
The possibility of direct writing thin semiconductive channels and structures on insulating substrates in a clean room-free process is attractive for its simplicity, cost effectiveness and possibility of a wide choice of substrates. A broad range of applications, such as large-area electronic devices (touch screens, flexible displays), sensors, or optical wave guides could benefit from such a process. In this work we directly write on doped hydrogenated amorphous silicon (a-Si:H), with thickness in the range 10 $\mathrm{nm}$ to $1 \mu \mathrm{m}$, using a Nd-YAG laser operating at $532 \mathrm{~nm}$ that is part of a Witec Raman confocal system. The contrast in conductivity between the exposed and unexposed areas is so high that the a-Si:H matrix needs not to be removed after exposure.

B- and P-doped films were deposited on plastic, glass, and oxidized silicon wafers. The laser power threshold for crystallization was studied. The highest conductivity (886 $\Omega^{-1} \mathrm{~cm}^{-1}$ ) was obtained on wafer. On hard substrates, it is possible to tune the mesoscopic electrical conductivity in a very broad range of values $\left(\sim 10^{-4}-10^{3}\right)$ by design of the
\end{abstract}

1 Introduction The possibility of direct writing thin semiconductive channels or structures on insulating substrates in a clean room-free process is attractive for its simplicity, cost effectiveness and wide choice of substrates allowed, including flexible plastics. A broad range of applications, such as optical wave guides, touchscreens, flexible displays, and other large-area electronic devices could benefit from such a process.

Hydrogenated nanocrystalline silicon (nc-Si:H) thin film deposition by CVD methods usually entails incubation layers that are tens of nanometers thick, with both very low conductivity and crystalline fraction. Doping such films is virtually impossible, because of the low-doping efficiency of amorphous silicon combined with the increased defect pattern to be transferred. Patterned films are piezoresistive with gauge factors as high as +18 and -29 for $p$ - and $n$-type patterns, respectively.

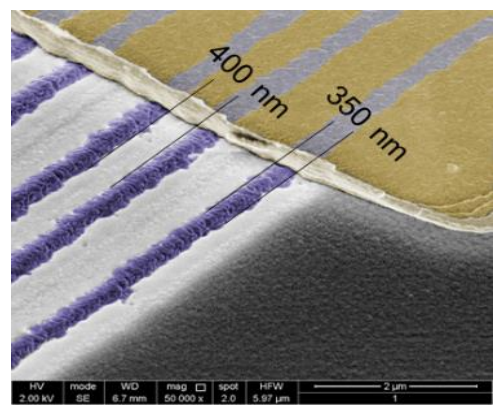

SEM image of laser written lines on a $10 \mathrm{~nm}$ thick a-Si:H film deposited on a $\mathrm{SiO}_{2} / \mathrm{Si}$ substrate. Four regions are clearly distinguishable: the metal contact on the top area; the laser eroded area (lines); the crystallized areas adjacent to lines; the amorphous region at the bottom right.

Copyright line will be provided by the publisher

density found in these layers, a consequence of lattice mismatch at the film-substrate interface. This transitional incubation zone is observed in all commonly used substrates, such as glass, plastic or stainless steel [1-3], using standard deposition methods, such as radio-frequency plasma-enhanced chemical vapor deposition (RF) or hot-wire CVD (HW). In previous works we succeeded to crystallize and activate the dopants of very thin $(60-100 \mathrm{~nm})$ doped hydrogenated amorphous $\mathrm{Si}(\mathrm{a}-\mathrm{Si}: \mathrm{H})$ layers deposited on glass and on plastic substrates using the $193 \mathrm{~nm}$ line of an excimer-laser (ArF) to irradiate the samples [4]. However this approach failed in case of film thickness lower than $60 \mathrm{~nm}$, because of irreversible damage inflicted to the substrates. In 
the present work, a frequency-doubled Nd:YAG laser operating at $532 \mathrm{~nm}$ is used to successfully write on a-Si:H films with thickness down to $10 \mathrm{~nm}$ deposited by RF and HW on plastic (polyimide) and on oxidized Si wafer substrates. The laser writing process consists of laser-induced silicon crystallization and dopant activation (P or B atoms for $n$ - and $p$ type doping, respectively) intentionally added to the gas phase during deposition of the amorphous Si writing medium.

The contrast in conductivity between the laser crystallized areas and the a-Si:H background is so high that the process works as a lithographic tool to write doped crystalline conductive channels without the need to etch away the remaining unexposed amorphous film covering the substrate. For example, starting with a $10 \mathrm{~nm}$ thick a-Si:H film deposited on polyimide (deposition time $=15 \mathrm{~s}$ ) from a gaseous mixture of $\mathrm{SiH}_{4}, \mathrm{H}_{2}$ and $\mathrm{B}_{2} \mathrm{H}_{6}$, an increase in dark conductivity from $\sim 10^{-7}$ to $63 \Omega^{-1} \mathrm{~cm}^{-1}$ was achieved using a 3.8 $\mathrm{mW}$ laser power irradiated through a $50 \times$ objective lens. Writing on a $40 \mathrm{~nm}$ a-Si:H gas phase doped with $\mathrm{PH}_{3}$ deposited on a $\mathrm{Si} / \mathrm{SiO}_{2}$ wafer substrate, after a $40 \mathrm{~mW}$ laser irradiation through a $100 \times$ objective lens, a dark conductivity of $886 \Omega^{-1} \mathrm{~cm}^{-1}$ was achieved.

Using the software that we developed to generate $2 \mathrm{D}$ patterns [5], it is relatively simple to tune the effective conductivity of the irradiated areas in a broad range of conductivity values by selecting the appropriate pattern (lines, dashes or dots, and the horizontal and vertical distances between them) and other writing parameters (e.g., scan speed and duty cycle), without changing the laser continuous output power. Electrical conductivity in a range between $\sim 10^{-4}$ and $850 \Omega^{-1} \mathrm{~cm}^{-1}$ was obtained. Moreover, the irradiated films are piezoresistive, i.e., their conductivity changes when deformed by strain. Measurements of the gauge factors $(G F)$ - the relative resistance change per unit of applied strain - showed that $p$-type films have positive longitudinal gauge factors and that $n$-type films have negative $G F \mathrm{~s}$.

\section{Experimental}

2.1 Thin film deposition Films were deposited in a ultra-high vacuum (base pressure $\sim 10^{-7}$ Torr) twin chamber system, one for RF and the other for HW. Substrates used were $600 \mu \mathrm{m}$ silicon wafers insulated with a $200 \mathrm{~nm} \mathrm{S^{2 }}{ }_{2}$ film, $1 \mathrm{~mm}$ thick borosilicate glass, and flexible polyimide (PI) and polyethylene terephthalate (PET) plastic substrates, $175 \mu \mathrm{m}$ thick. Film thickness was in the range $10 \mathrm{~nm}$ to 1 $\mu \mathrm{m}$. Dopant gases $-\mathrm{PH}_{3}$ and $\mathrm{B}_{2} \mathrm{H}_{6}$, for $n$ - and $p$-type doping, respectively - were added to the reactive gaseous mixtures of $\mathrm{SiH}_{4}$ and $\mathrm{H}_{2}$ in a manifold and then injected into the CVD chamber for film growth. Dopant gas-to- $\mathrm{SiH}_{4}$ flow rate ratio was kept constant at $2 \%$ for all depositions. Substrate temperature was in the range from 150 to $220^{\circ} \mathrm{C}$. Working pressure was 40 mTorr for HW depositions and was varied from 100 to 1500 mTorr in case of RF depositions. Filament temperature was $\sim 2000{ }^{\circ} \mathrm{C}$ for $\mathrm{HW}$ growth and rf-power density was varied from 50 to $250 \mathrm{~mW} / \mathrm{cm}^{2}$. Although deposition conditions varied a lot (see Table 1) the parameter range used both for HW and for RF depositions was chosen in such a way as to keep the hydrogen content in the a-Si:H films low. This was essentially done by keeping the $\mathrm{H}_{2}$ dilution of the reactive gases $\left(D_{\mathrm{H}}\right)$, defined as the $\mathrm{H}_{2}$ flow divided by the total gas flow times $100 \%$, relatively high (although below the threshold value for nanocrystalline phase growth): for $\mathrm{HW}, D_{\mathrm{H}}$ was in the range $60-90 \%$, and for RF, $D_{\mathrm{H}}$ was in the range $90-96 \%$. As will be shown in the next sections, there was no significant influence of varying the deposition conditions on the properties of the films after laser irradiation.

Table $1 \mathrm{CVD}$ parameters used for a-Si:H depositions.

\begin{tabular}{lcc}
\hline Deposition parameter & HW & \multicolumn{2}{c}{ RF } \\
\hline $\begin{array}{l}\text { Substrate temperature, } T_{\text {sub }}\left({ }^{\circ} \mathrm{C}\right) \\
\text { Working pressure, } p_{\mathrm{w}}(\mathrm{mT} \text { Torr })\end{array}$ & \multicolumn{2}{c}{$150-220$} \\
$\begin{array}{l}\text { Filament temperature, } T_{\text {fil }} \\
\text { rf-power density }\left(\mathrm{mW} / \mathrm{cm}^{2}\right)\end{array}$ & $1750-2000$ & $100-500$ \\
$\begin{array}{l}\text { Substrate-to-filament, or inter- } \\
\text { electrode distance, } d_{\text {fil }} \text { or } d_{\mathrm{i}-\mathrm{e}}\end{array}$ & 7 & - \\
$\begin{array}{l}(\mathrm{cm}) \\
\text { Hydrogen dilution, } D_{\mathrm{H}}(\%)\end{array}$ & $60-1500$ \\
\hline
\end{tabular}

2.2 Laser writing set up The lithography set up consists of the following elements: (i) a Nd:YAG frequencydoubled $532 \mathrm{~nm}$ laser; (ii) an electronically controlled mechanical shutter mounted in front of the laser beam; (iii) a microscope with Zeiss objective lenses EC Epiplan 50X $(\mathrm{NA}=0.7)$ and $100 \mathrm{X}(\mathrm{NA}=0.9)$; (iv) a single mode optical fiber connecting the laser to the microscope; (v) a motorized $\mathrm{XY}$-sample stage with a positioning accuracy better than 1 $\mu \mathrm{m}$ and a displacement range of $2.5 \mathrm{~cm}$ both in $\mathrm{X}$ and $\mathrm{Y}$ directions; and (vi) a computer running the Witec Control Four software, driving the shutter, the motorized stage, and the z-distance between the microscope objective and the writing medium. The Witec Control Four software accepts text files containing instructions that open/close the shutter, move the stage (position and velocity) and the objective lens vertically, according to the pattern to be transferred. Therefore, we developed in house a software that reads most kinds of drawing programs (e.g. AutoCad) and converts the readings into a text file containing Witec compatible instructions In this way any drawings created in different software environments can be transferred onto the writing medium. Our computer program can also generate periodic patterns of any shape based on lines, dashes and dots, in the form of a set of instructions for the Witec Control Four software. In this study, in all cases the films were irradiated in rectangular patterns, consisting of parallel lines or dashes with different separations between lines and different duty cycles, in case of dashes, which were generated with our program. Figure 1a) shows a scheme of the writing setup. 


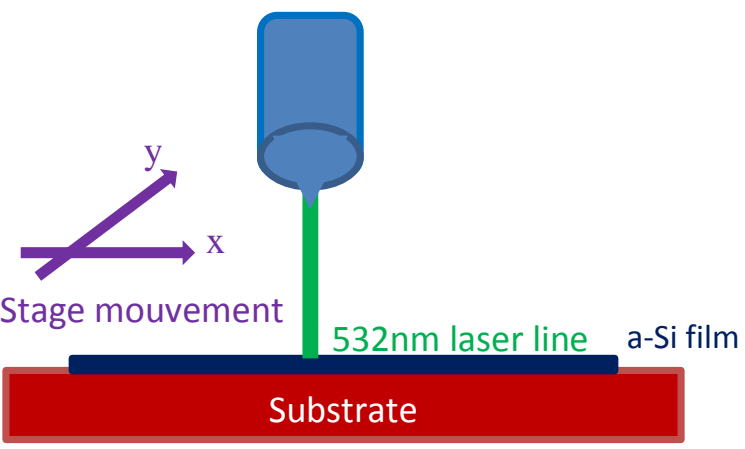

Figure 1 Scheme of the laser writing set up.

2.3 Thin-film characterization The irradiated areas were studied by Raman spectroscopy, Scanning Electron Microscopy (SEM) and Conductive Atomic Force Microscopy (C-AFM) in order to elucidate the structure and crystallization mechanisms. Dark conductivity measurements were performed between parallel $\mathrm{AlSiCu}$ sputtered contacts defined using either a shadow mask of standard lithography.

The Raman study was performed, before and after patterning, using very low laser power $(<0.5 \mathrm{~mW})$, to ensure that no crystallization is induced at this stage. Raman crystalline fraction $\left(X_{\mathrm{c}}\right)$ and average crystallite size $\left(d_{\text {Raman }}\right)$ are calculated according to reference [8]. C-AFM working in contact mode, using a Pt/Ir coated tip, was employed to correlate topography and electrical properties at the nanoscale. Current maps were obtained by applying a constant voltage to the sample while the (grounded) tip scanned the area under analysis. Topography and conduction maps of the scanned area are obtained simultaneously with this technique.

\section{Results and discussion}

3.1 Study of the laser crystallization process In this section we report on the systematic study of direct laser writing by crystallization and dopant activation of a-Si:H thin films. The first step was to establish the laser power threshold for light-induced crystallization of the films, which proofed to be very different for different types of substrates. In contrast, we observed no difference in the laser fluence for crystallization required for $n$ - and $p$-type films. In general, the laser power threshold for crystallization increases with the thermal conductivity of the substrate. It also depends on the lens used and on the writing speed, which, together with the laser power, sets the fluence. Using a 100x lens at a scan speed of $62 \mu \mathrm{m} / \mathrm{s}$, the threshold for crystallization was 4, 7 and $15 \mathrm{~mW}$, for PI and PET, glass, and c-Si substrates, respectively. The power threshold for crystallization was determined by irradiating the films with increasing laser power, keeping other parameters fixed, followed by micro-Raman analysis of the irradiated areas, until the amorphous-to-nanocrystalline phase transition was detected. In the range of film thickness studied $(10-1000 \mathrm{~nm})$ the threshold for crystallization does not depend on the film thickness.

A SEM systematic study of the energy threshold for crystallization and of the structure of the irradiated areas, as a function of radiant fluence (laser power $x$ irradiation time) was done. To this goal, a matrix of "dots" was statically irradiated using a $100 \times$ lens on a $10 \mathrm{~nm}$ thick $\mathrm{p}^{+}$-a-Si:H film deposited on an insulating layer of $\mathrm{Al}_{2} \mathrm{O}_{3}(260 \mathrm{~nm})$ on a $\mathrm{Cu}$ substrate (Fig. 2). The Cu foil ( $25 \mu \mathrm{m})$ was used as substrate for better SEM image quality, avoiding charge accumulation in the sample that blurs the image. Each line of the matrix corresponds to a fixed laser power $(30,33,35$, $40 \mathrm{~mW}$ ) and each column corresponds to an irradiation time $(0.09,0.9,9,45 \mathrm{~s})$. Compared with other substrates, the threshold for crystallization is higher in case of the $\mathrm{Cu}$ foil, which is understandable in view of the higher thermal conductivity of copper. The diameter of the crystallized area $(\sim 1 \mu \mathrm{m})$ is of the same order of magnitude of the laser spot. Some samples show concentric rings of varying contrast similar to an Airy pattern. This happens only for the longest exposure times and is probably due to transfer of the

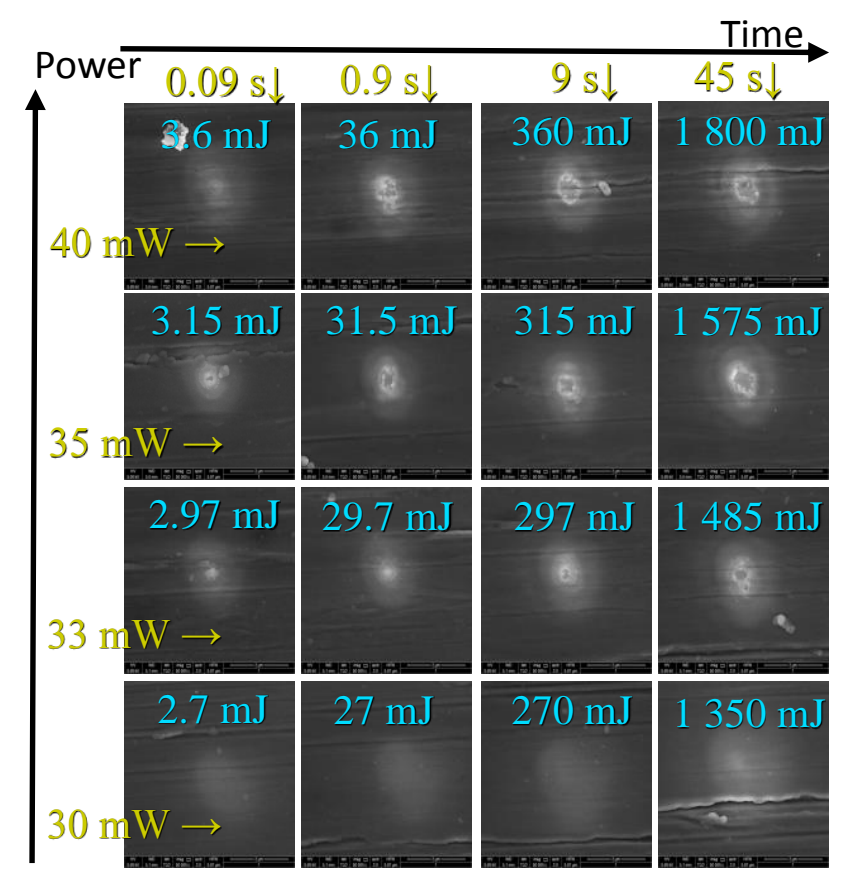

Figure 2 - SEM images of laser statically irradiated "dot" matrices with varying laser power (lines) and exposure time (columns). The scale bar is $2 \mu \mathrm{m}$ in all micrographs.

diffraction pattern produced by the lens with a circular aperture onto the a-Si:H film [6]. For laser power > $30 \mathrm{~mW}$ ablation of the Si film occurs at the center of the crystallized area. Fig. 3 shows magnified images of two similarly ablated areas resulting from irradiation of a $40 \mathrm{~nm}$ a-Si:H film deposited on a Si/SiO2 substrate at a laser power of $40 \mathrm{~mW}$. Several different regions can be identifyied in Fig.3a): (i) the circular, volcano-like black spot, with diameter $80 \mathrm{~nm}$ is 

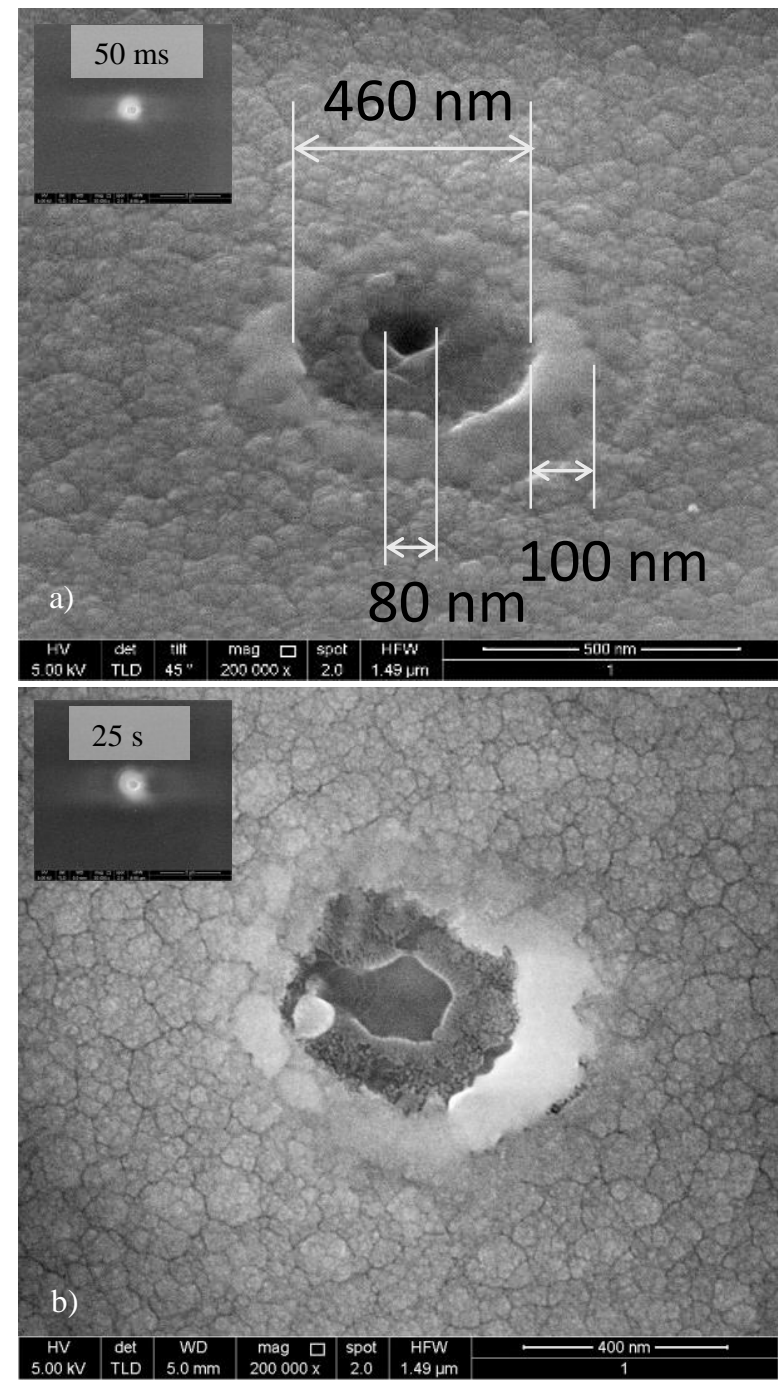

Figure 3 SEM images of statically irradiated areas using a $100 \times$ objective lens and $40 \mathrm{~mW}$ laser power during a) $50 \mathrm{~ms}$ and, b) $25 \mathrm{~s}$. The scale bar in the insets is $3 \mu \mathrm{m}$.

centered on the laser spot, around the image plane of the optical set up. This area is the most severely heated, where explosive crystallization and ablation occurs. (ii) A circular dark grey area with diameter $460 \mathrm{~nm}$ with a non-uniform coverage of recrystallized silicon (droplets of crystallized $\mathrm{Si}$ ). (iii) A $100 \mathrm{~nm}$ wide annular border with lighter contrast, crystallized with very fine grain. This is the area of accumulation where most of the melted silicon ablated from under the center of the beam accumulates and solidifies. 4) The remaining area, extending beyond the region pictured (but seen in the inset, with diameter $\sim 2 \mu \mathrm{m}$ ) is silicon, placed close to the image plane of the optical system, which is crystallized by the laser beam, far from its center. The difference between Fig.3a) and b) is the exposure time, which was $50 \mathrm{~ms}$ and $25 \mathrm{~s}$, respectively. The structure of the interaction area is similar in a) and b) except that the central area is not a tiny black spot, but a larger $(120 \mathrm{~nm})$ area, from where the Si film was completely ablated. Similar results were obtained on $n$-type films.

Fig. 4 shows SEM images at a lower magnification of two dots representative of the two regions discussed in relation with Fig. 3a) and 3b). The first dot, in 4a), was written at a low fluence ( $30 \mathrm{~mW}$ for $1 \mathrm{~s})$, and the second one, in $4 \mathrm{~b}$ ), at a high fluence ( $35 \mathrm{~mW}$ for $45 \mathrm{~s}$ ). Only the latter shows vestiges of explosive crystallization, under the center of the beam. At this magnification, the bright contrast of the laser exposed area can be seen to extend radially up to a circle that is $\sim 3 \mu \mathrm{m}$ in diameter, in both images. In fact, its size slightly increases with laser fluence, from $\sim 2.5$ to $3 \mu \mathrm{m}$ in diameter, from a) to b). In Fig.4b), the outer limits of those two areas (melted and crystallized, respectively) were marked by two arcs of circle. Inside the melted area the temperature during process can be estimated to be Si melting temperature $(1687 \mathrm{~K})$. As stated above, its size is comparable to the diameter of the laser beam. To better understand the size of the bright area, let us consider a very simple model, which invokes the heat equation in cylindrical coordinates. In the model, we consider that the laser energy incident on the material is transferred laterally to the surrounding a-Si:H writing medium, within a certain thickness. It is convenient to remind that the sample consists of $40 \mathrm{~nm}$ of a-Si:H on top of $260 \mathrm{~nm} \mathrm{Al}_{2} \mathrm{O}_{3}$ on $\mathrm{Cu}$. For simplicity, we will consider only one material (silicon), and treat the heat transfer as a transfer within an effective Si thickness. Referring to the two concentric areas in Fig.4b), the first has radius $r_{1}$, as measured in Fig.3a) $\left(r_{1}=460 / 2 \mathrm{~nm}\right)$, sets a limit for the molten silicon region, at a temperature $T_{l}$ $=1687 \mathrm{~K}$. Let us arbitrarily define a radius where we can state safely that the temperature of the material was not significantly changed during the process, say $r_{2}=5 \mu \mathrm{m}$ where $T_{2}=300 \mathrm{~K}\left(=r_{\infty}\right)$. A steady state solution to the heat conduction equation for these two limiting temperatures, is:

$$
T(r)=T_{2}+\left(T_{1}-T_{2}\right) \times \frac{\ln \left(r_{2} / r\right)}{\ln \left({ }^{r_{2}} / r_{1}\right)}, \text { for } r_{1}<r<r_{2}
$$
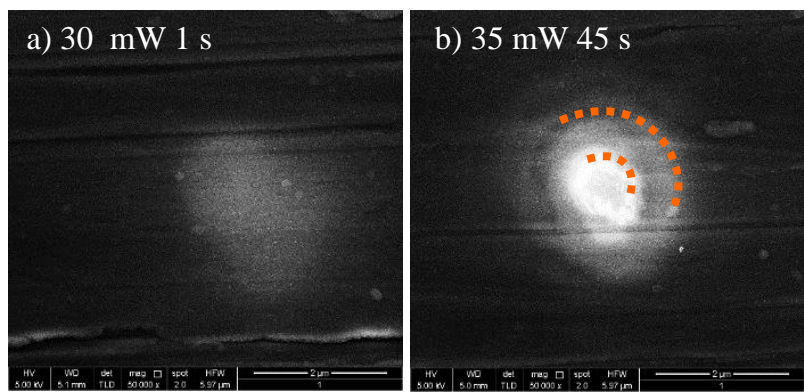

Figure 4 - SEM image of a dot exposure for two particular conditions: $30 \mathrm{~mW}, 1 \mathrm{~s}$ (left) and $35 \mathrm{~mW}, 45 \mathrm{~s}$. The two images were obtained with the same brightness and contrast settings. On the dot exposed with $35 \mathrm{~mW}$, where silicon melting happened, two arcs of circles are overlaid as a guide for the eye. The inner circle $\left(r_{\text {in }} \approx 0.4 \mu \mathrm{m}\right)$ points out the edge of the melted + accumulation areas. The outer circle $\left(r_{\text {out }} \approx 1.5 \mu \mathrm{m}\right)$ points out the limit of significant SEM contrasted area. 
Eq.1 predicts that the temperature will decrease logarithmically from the edge of the melted area, where temperature is $T_{l}$, in to the region where the material is considered unaffected by the laser. Substituting for the values of $T_{1}, T_{2}$, $r_{1}, r_{2}$, and interrogating for the temperature at radius $r_{\mathrm{out}}=$ $1.5 \mu \mathrm{m}$, which in average sets the limit of the bright region (see Fig.4b)), Eq.1 gives $T \approx 840 \mathrm{~K}$, which is close to the temperature at which amorphous silicon undergoes a solidstate transition from amorphous to microcrystalline [REF]. This result is consistent with the interpretation that the edge of the SEM contrasted area corresponds to the end of the crystallized, highly conductive zone.

In the same geometry, the solution to the heat equation can also be expressed by a flux transferred between the regions defined by the two concentric radii: $T(r)=T_{2}+\left(q_{1} r_{1}\right.$ / $k) \times \ln \left(r_{2} / r\right)$, with $k=148 \mathrm{~W} \mathrm{~m}^{-1} \mathrm{~K}^{-1}$ the thermal conductivity of silicon and $q_{1}$ the flux through a cylindrical surface at a distance $r_{1}$ from the heat source. Assuming that the heat transport is by conduction and that the flow is radially symmetric in horizontal planes centered at the laser spot, the flux $q_{1}=P /\left(2 \pi r_{1} h^{*}\right)$ where $P$ is laser power and $h^{*}$ is Si effective thickness. Solving for $h^{*}$ with $T\left(r_{1}\right)=T_{1}$, the effective Si thickness can be evaluated, giving $h^{*}=80 \mathrm{~nm}$ (i.e., twice the film thickness). The larger value of $h^{*}$ as compared to the film thickness stems from the incomplete absorption of the laser beam in the a-Si:H film, and accounts for the lateral heat transfer in the underlying layers.

SEM images of $p$-type silicon laser-crystallized lines, written every $\sim 1.2 \mu \mathrm{m}$, on a $\mathrm{Si} / \mathrm{SiO}_{2}$ substrate at $40 \mathrm{~mW}$ laser power and $62 \mu \mathrm{m} / \mathrm{s}$ scan speed are shown in Fig.5a) and b).
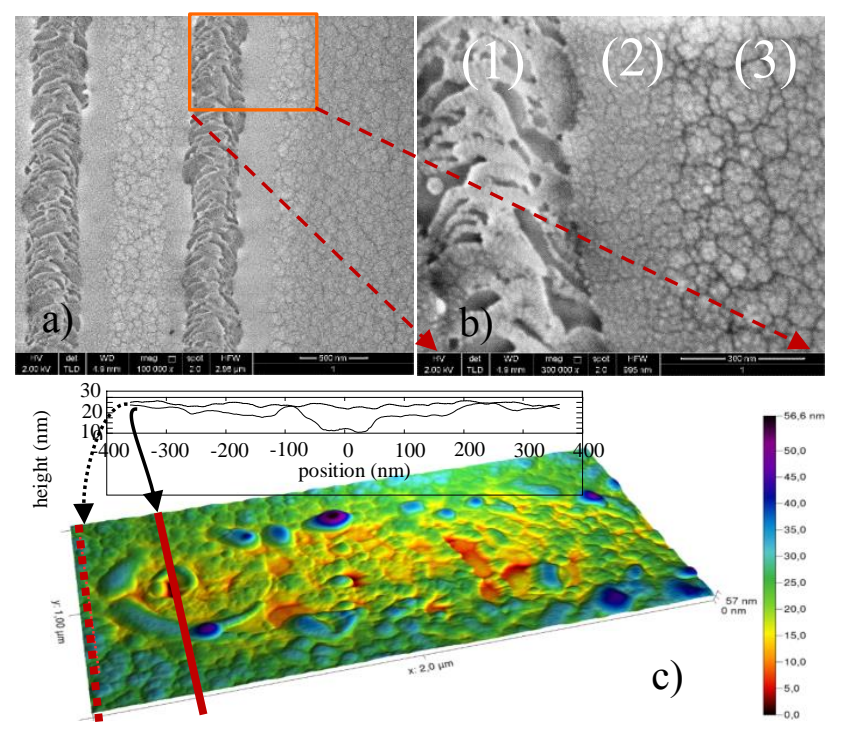

Figure 5 - Morphology of laser-written lines observed by SEM. (a) Top view. (b) Top view under higher magnification of the area inside the rectangle in (a). The numbers (1) to (3) correspond to the regions discussed in the text. c) AFM profiles of two transverse line scans: the first (dotted line) outside the exposed area. The second (solid line) over a cross section of the laser path.
The same structural features and distinctive areas that were found for static irradiation are also present here. It is possible to identify in Fig.5a) and b) three different film morphologies, namely: region (1), the path of the center of the laser beam (350-400 $\mathrm{nm}$ wide), in which the amorphous silicon writing medium melts and is partially ablated. The laser beam movement was in the sense from bottom to top in the pictures; region (2) is a crystallized area upon solidification of the melted silicon. Region (3) is an area that has undergone solid-phase crystallization, with crystallite size larger than in region (2) but still of nanometric size $(6-8 \mathrm{~nm}$ as measured by Raman). The total width of the laser crystallized line is estimated to be $1.2 \mu \mathrm{m}$, since adjacent crystallized lines coalesce. This is similar to the diameter of the crystallized area in static mode. In Fig.5c) AFM profiles of two transverse line scans, outside (dotted line) and inside (solid line) the laser path at the place where the laser lithography started, are displayed. A circular area is located at the place where the laser lithography started. The ablated spot is visible in the center. The spot has a depth comparable to that of the amorphous silicon layer, confirming that the film was completely ablated at this laser power $(40 \mathrm{~mW})$.

In Fig. 6 the effect of increasing the laser power on the crystallite size and crystalline fraction measured by Raman spectroscopy, while keeping all other parameters constant (scan speed $=62 \mu \mathrm{m} / \mathrm{s}$, lens 50x), is seen for $n$-type samples deposited on a glass substrate. Whereas the crystallite size remains almost unchanged $(\sim 6.5-7 \mathrm{~nm})$, the crystalline fraction increases from $63 \%$ at the power threshold for crystallization ( $7 \mathrm{~mW}$ ), up to $96 \%$ at $28 \mathrm{~mW}$ laser power, in this particular set of measurements.

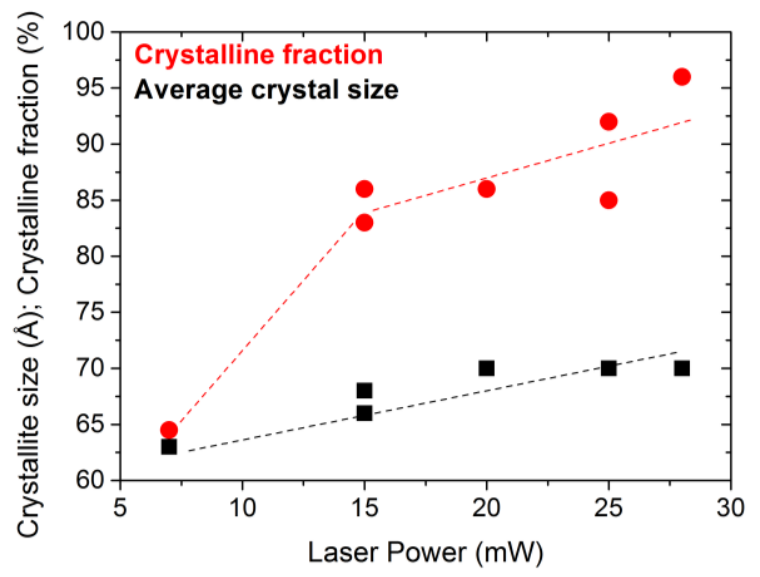

Figure 6 Crystalline fraction and crystallite size measured by Raman spectroscopy as a function of laser power (scan speed $=62 \mu \mathrm{m} / \mathrm{s}$, lens 50x, substrate: glass) (dashed lines are mere guides to the eye). 
3.2 Patterning at the mesoscopic scale After the previous study, where the morphology and dimensions of the laser-film interaction area were established, it is clear that, for crystallization of a macroscopic region on a substrate, even small, e.g., an area with some tens or hundreds of micrometers in length and width, it is necessary to laserscan the sample in a systematic way, until the desired region is crystallized. It involves the division of the area to be irradiated in smaller domains, e.g., rectangles that will be successively scanned by the laser. This is done with the help of the software program that we developed, which generates any type of mesh to fill the closed regions that result from the decomposition of drawings imported from common drawing programs, into a set of instructions that the Witec lithography tool will understand. Our software program can also analytically generate arrays of geometric motifs with any shape to fill any given area. The next step in our study was, therefore, to see the effect of the scan path, and of the segmentation of an area, on the macroscopic properties of the resulting crystalline region, namely its effective electrical conductivity. To that end, several periodic patterns were laser scribed on the films over similar areas of $100 \times 80 \mu \mathrm{m}^{2}$. The patterns were: A) $100 \mu \mathrm{m}$ long parallel lines with interline spacing of: i) $1 \mu \mathrm{m}$; ii) $2 \mu \mathrm{m}$; iii) $3 \mu \mathrm{m}$; and iv) $5 \mu \mathrm{m}$; B) dashed lines with dash length $2 \mu \mathrm{m}$ and inter-line spacing of 1 and $2 \mu \mathrm{m}$. The duty cycle (the fraction of time that the laser shutter is open over an entire line), which sets the distance between dashes along the line, was also varied: (i) $95.2 \%$; (ii) $90.9 \%$; (iii) 80 ; (iv) $66.7 \%$; and (v) $50 \%$. Patterns B)-(i) to (v) were repeated with dashes between adjacent lines placed in- and out-of-phase. The patterns were written at two different scan speeds, namely $15 \mu \mathrm{m} / \mathrm{s}$ and 62 $\mu \mathrm{m} / \mathrm{s}$, for a set of laser powers, giving rise to a multiplicity of replicas of the same pattern geometry obtained under different combinations of writing parameters. Table 2 shows microscope images of selected samples where the same pattern was written on $\mathrm{p}^{+}$-a-Si:H deposited on polyimide (left column) and on $n^{+}-\mathrm{a}-\mathrm{Si}: \mathrm{H} \mathrm{Si} / \mathrm{SiO}_{2}$ wafer (right column). In both cases the film thickness was $40 \mathrm{~nm}$. The dark conductivity of each region, measured between parallel $\mathrm{AlSiCu}$ contacts, is given for each structure (current flow was in the horizontal direction, referred to the images). The patterns were written at a fluence of $15 \mathrm{~mW}(40 \mathrm{~mW})$ at $62 \mu \mathrm{m} / \mathrm{s}$ scan speed for the film deposited on polyimide (wafer) substrate. The higher power used for the wafer substrate is a consequence of the higher threshold power for crystallization in this case.

Fig.7 shows the Raman spectra of the samples displayed in Table 2. The Raman crystalline fraction, $X_{\mathrm{c}}$, strongly depends on the pattern written on a-Si, particularly on $\mathrm{Si} / \mathrm{SiO}_{2}$ substrates, which dissipate heat much faster than PI. This allows for a very precise pattern transfer. For this reason, on $\mathrm{SiO}_{2}$ substrates any crystalline fraction in the range 7-100\% can be virtually obtained by playing with interline separation, duty cycle of dashed lines, and other geometric parameters in the pattern (see Table 2 and
Table 2 Patterns written on a-Si:H deposited on polyimide and on $\mathrm{Si} / \mathrm{SiO}_{2}$ wafer substrates and their dark conductivity

\begin{tabular}{|c|c|c|}
\hline \multirow{2}{*}{$\begin{array}{l}\text { Written } \\
\text { pattern }\end{array}$} & \multicolumn{2}{|c|}{ Writing medium } \\
\hline & a-Si on Polyimide & $\begin{array}{l}\text { a-Si on } \mathrm{SiO}_{2} / \mathrm{Si} \\
\text { wafer }\end{array}$ \\
\hline $\begin{array}{l}\text { L1 } \\
\text { Lines- } \\
2 \mu \mathrm{m} \\
\text { spaced }\end{array}$ & 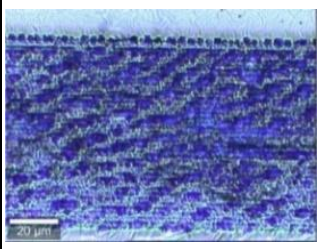 & \\
\hline & $\sigma_{d}=60 \Omega^{-1} \mathrm{~cm}^{-1}$ & $\sigma_{d}=426 \Omega^{-1} \mathrm{~cm}^{-1}$ \\
\hline $\begin{array}{l}\text { L2 } \\
\text { Horizon } \\
\text { tal } \\
\text { dashes } \\
\text { in phase } \\
-2 \mu \mathrm{m}\end{array}$ & 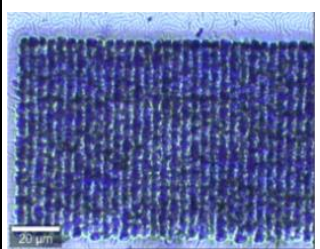 & \\
\hline & $\sigma_{d}=46 \Omega^{-1} \mathrm{~cm}^{-1}$ & $\sigma_{d}=58 \Omega^{-1} \mathrm{~cm}^{-1}$ \\
\hline $\begin{array}{l}\text { L3 } \\
\text { Hori- } \\
\text { zontal } \\
\text { dashes } \\
\text { out of } \\
\text { phase - }\end{array}$ & 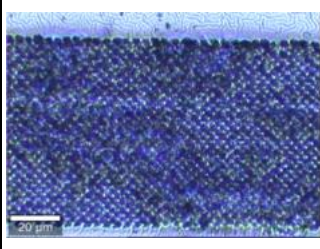 & \\
\hline $2 \mu m$ & $\sigma_{d}=60 \Omega^{-1} \mathrm{~cm}^{-1}$ & $\sigma_{d}=31 \Omega^{-1} \mathrm{~cm}^{-1}$ \\
\hline $\begin{array}{l}\text { L4 } \\
\text { Vertical } \\
\text { dashes- } \\
2 \mu \mathrm{m}\end{array}$ & 25 & 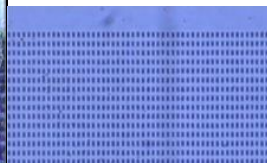 \\
\hline & $\sigma_{d}=52 \Omega^{-1} \mathrm{~cm}^{-1}$ & $\sigma_{d}=37 \Omega^{-1} \mathrm{~cm}^{-1}$ \\
\hline
\end{tabular}
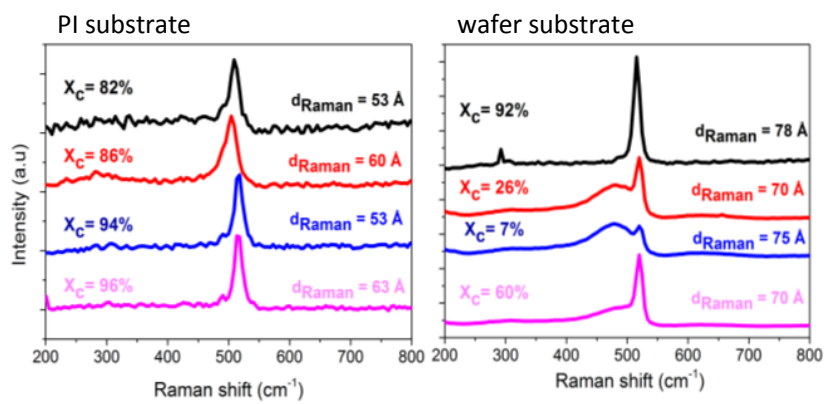

Figure 7 Raman spectra of the films and patterns reported in Table 2 .

Fig.7), i.e. by changing the geometry while keeping all laser-writing parameters unchanged. 
The ability to control the crystalline fraction of these patterns is the mesoscopic equivalent of controlling the crystalline volume fraction of $\mathrm{nc}-\mathrm{Si}: \mathrm{H}$ at the microscopic scale, a key parameter for many applications [7]. Like in the microscopic case, the electrical conductivity of the mesoscopic samples is correlated with $X_{\mathrm{C}}$. However, in the patterned samples, the crystalline phase is distributed in a periodic geometric pattern transferred onto the films. This introduces a new parameter that distorts the usual correlation between structure and conductivity observed in directly deposited nc$\mathrm{Si}: \mathrm{H}$ because the crystallized area can be arranged in ways that favor more, or less, the percolation of charge carriers between the metallic contacts. For example, looking at Table 2 one sees that, on $\mathrm{Si} / \mathrm{SiO}_{2}$ substrates, the same crystallized area with two different spatial arrangements, namely horizontal $2 \mu \mathrm{m}$ dashes, in and out of phase (lines 2 and 3 , respectively), produces conductivities that roughly are one twice the other $\left(\sigma_{\mathrm{d}}=58\right.$ and $31 \Omega^{-1} \mathrm{~cm}^{-1}$, respectively). By rotating the pattern $90^{\circ}$ (vertical dashes $2 \mu \mathrm{m}$ apart, line 4) one obtains $\sigma_{\mathrm{d}}=37 \Omega^{-1} \mathrm{~cm}^{-1}$. The pattern consisting of lines, with line separation of $2 \mu \mathrm{m}$, gives $\sigma_{\mathrm{d}}=426 \Omega^{-1} \mathrm{~cm}^{-1}$ and $X_{\mathrm{C}}$ $=92 \%$, which is the highest value obtained for the samples in Table 2. The highest value of conductiviy obtained in the present study was $\sigma_{\mathrm{d}}=886 \Omega^{-1} \mathrm{~cm}^{-1}$ on a $n$-type film on $\mathrm{Si} / \mathrm{SiO}_{2}$ substrate, using a pattern of lines with line separation $0.5 \mu \mathrm{m}$, and the same writing conditions that were used for the samples in Table 2 . The writing medium was, however, thicker (a-Si:H thickness $d=150 \mathrm{~nm})$. The measured crystalline fraction was $X_{\mathrm{C}}=98 \%$, which shows that, under the writing conditions used, the crystallized regions from adjacent lines essentially overlap. For compari- son, a sample of monocrystalline $\mathrm{Si}$ with the same conductivity would require a P-doping level of $\sim 6.9 \times 10^{19} \mathrm{~cm}^{-3}$ and would have a carrier mobility $\mu_{e}=80.5 \mathrm{~cm}^{2} \mathrm{~V}^{-1} \mathrm{~s}^{-1}$ [8].

For polyimide substrates, there is a much lower level of control of the properties of the irradiated areas for two main reasons: first, the substrates are thermal isolators and therefore there is enough accumulation of thermal energy in the $\mathrm{Si}$ film for propagation of the crystallization front away from the laser scribing track, which blurs the pattern transferred; second, the substrates have a comparatively low working temperature and deform locally at the surface under laser exposure, which reduces definition and possibly hinders crystallization due to carbon addition to the silicon melt. Another issue with plastic substrates is the need to correct often the distance of the lens above the substrate in order to keep the laser focused on the a-Si:H film over macroscopic distances. This is a consequence of the warping of the plastic foil over long distances. We corrected for this effect by doing preliminary measurements in several points of the substrate of the vertical $z$-position of the lens above the XYstage to keep the beam in focus. From that set of $z$-values, corrections were extrapolated for every $n$ patterned lines ( $n$ $=1,2,3 \ldots$ depending on the pattern and amount of warping) and the corresponding $\Delta z$ adjustments were included in the software program.

According to Table 2 , crystallite size, $d_{\text {Raman }}$, is relatively independent of the pattern written and is slightly higher in

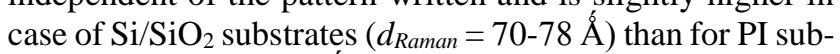

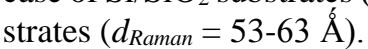

For a detailed study with nanoscopic resolution of the patterned areas we performed conductive AFM (C-AFM) measurements. For good image quality samples prepared
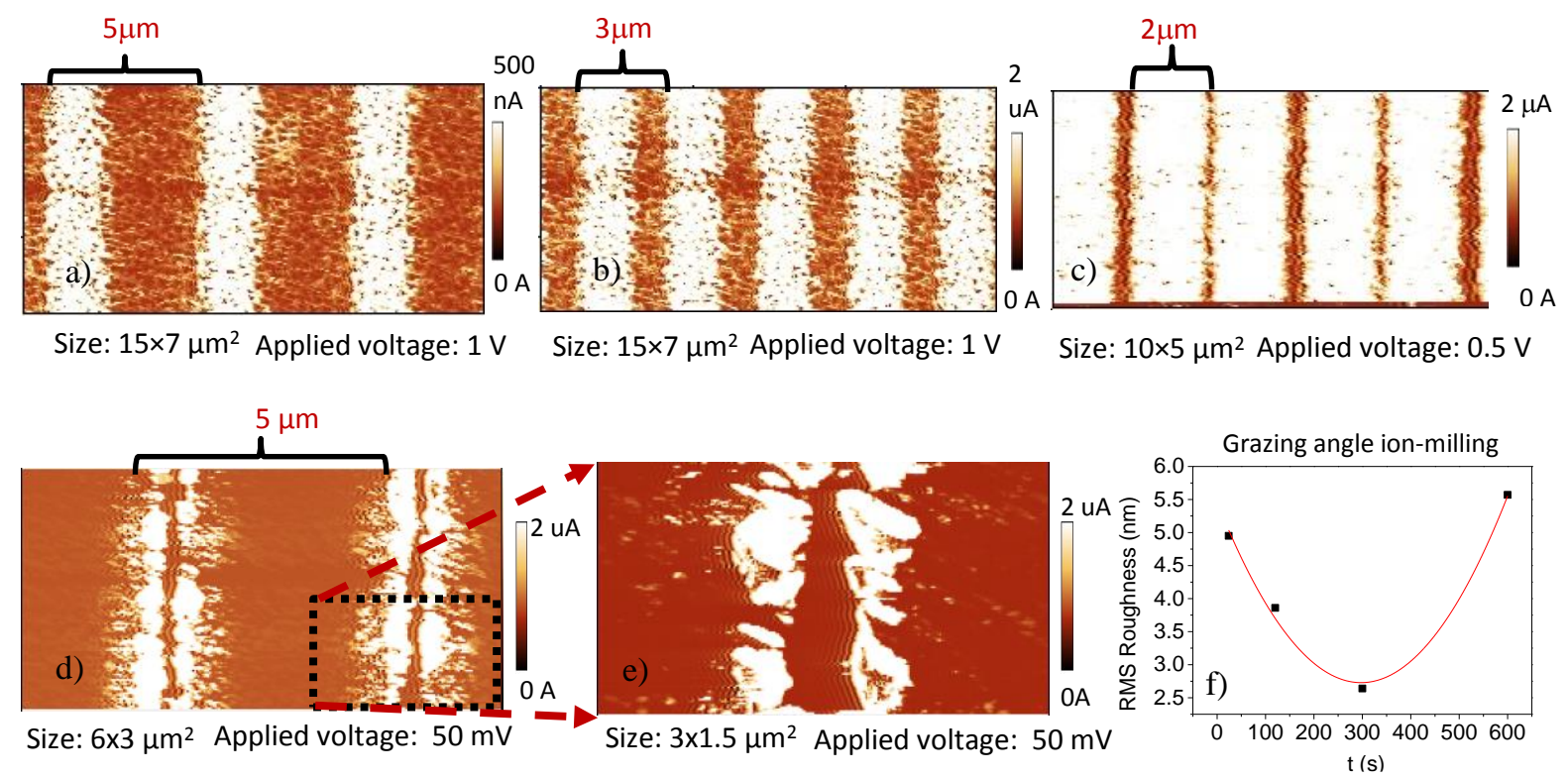

Figure 8 a) to c) - Conductive AFM images of samples patterned with lines of constant width ( $2 \mu \mathrm{m})$ but different interline spacing: 3, 1 and $0.5 \mu \mathrm{m}$, respectively. d) and e) - C-AFM images of lines written at a $40 \mathrm{~mW}$ and $3 \mu \mathrm{m}$ interline spacing. The analyzed area is identified bellow the images and the current scale is given in the vertical bar associated with the image. f) - RMS roughness as a function of time of grazing angle $\left(10^{\circ}\right)$ ion milling. 


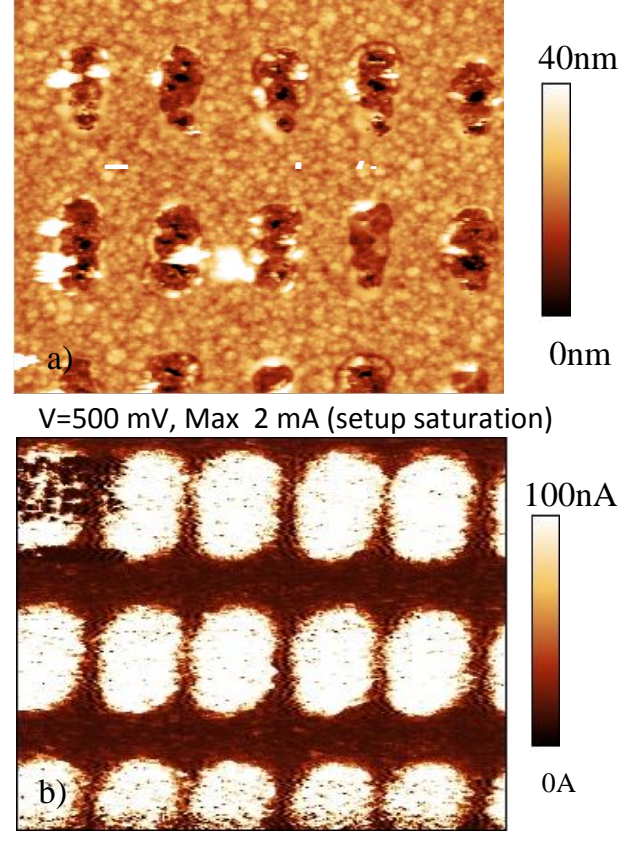

Size $10 \times 10 \mathrm{~mm} 2$

Figure 9 a) Contact mode topographical AFM image of a pattern consisting of vertical dashes $2 \mu \mathrm{m}$ long and equally spaced $(2 \mu \mathrm{m})$ in vertical and horizontal directions. b) Conductive AFM image of the same area.

specifically for these measurements are required: a $\mathrm{Si} / \mathrm{SiO}_{2}$ wafer was covered with a conductive layer of $\mathrm{TiW}(5 \mathrm{~nm})$, on top of which $40 \mathrm{~nm} \mathrm{n} \mathrm{n}^{+}$-a-Si:H were deposited. Before AFM measurements, samples had their surface planarized by grazing angle $\left(10^{\circ}\right)$ ion milling using an unfocused $\mathrm{Ar}^{+}$ beam accelerated at $1.5 \mathrm{kV}$ (see Fig.8f)). This step was necessary to avoid tip damage and to improve image quality. The RMS roughness was reduced from $\sim 5.5 \mathrm{~nm}$ in the asirradiated samples to $\sim 2.5 \mathrm{~nm}$ after planarization, using 5 min milling time. Fig. 8 and Fig. 9 show current maps for different sample-to-tip applied voltages. The C-AFM analyzed area is identified bellow the images and the current scale is given in the vertical bar associated with the image. Fig.8a) to c) show three C-AFM images of samples patterned with lines of constant width $(\sim 2 \mu \mathrm{m})$ but different interline spacing: 3,1 and $0.5 \mu \mathrm{m}$, respectively. Laser power was $30 \mathrm{~mW}$ and scan speed was $62 \mu \mathrm{m} / \mathrm{s}$. If one identifies the crystallized areas as the bright zones of the C-AFM image (high tip-to-sample currents) and the amorphous medium as the brown regions (low tip currents) one concludes that the process could resolve this geometry down to $500 \mathrm{~nm}$ separation between lines. Fig.8d) and e), which display C-AFM images of lines written at a $40 \mathrm{~mW}$ laser power and the same scan speed as before, confirms that for this fluence there is ablation of the central part of the lines, right below the center of the laser beam.

In Fig.9a) shows a contact mode topographical AFM image of a pattern consisting of vertical dashes $2 \mu \mathrm{m}$ long and equally spaced $(2 \mu \mathrm{m})$ in both vertical and horizontal directions. Fig.9b) shows a C-AFM image of the same area, obtained simultaneously. It can be seen that the high-conductivity crystallized areas in b) extend beyond the topographic elements in a), which correspond to the Si laser ablated features under the center of the laser beam. This type of structure is the same observed in the SEM study before, for high laser fluence (see section 3.1). Moreover, it can be seen in Fig.9b) that the crystallization front propagates faster in directions perpendicular to the movement of the laser spot than along the same line. In fact, in the horizontal direction the dashes almost coalesce whereas in the vertical direction there is a much larger gap $(\sim 1 \mu \mathrm{m})$ consisting of highly resistive a-Si:H tissue.

Fig. 10 shows $\sigma_{\mathrm{d}}$ as a function of inter-line spacing, for line-spacing $0.5,1$ and $2 \mu \mathrm{m}$, measured on two samples $-n$ and $p$-type, respectively - written with the same patterns that were used for C-AFM measurements. The back AlSiCu contact layer, however, was replaced by two parallel metal $(\mathrm{AlSiCu})$ contacts on top of each patterned area, for the measurement of $\sigma_{\mathrm{d}}$. Points in the graph are averages of four measurements, each one made on one of the four replicas of the same pattern. The error bars are sample standard deviations. It is evident that the same pattern written on $n$ type films is more conductive than on $p$-type films. This could be a consequence of higher electron mobility than hole mobility, in silicon. For example, at a doping level of $10^{18} \mathrm{~cm}^{-3}$ the electron mobility is 4 times higher than the hole mobility ( 800 vs $200 \mathrm{~cm}^{2} \mathrm{~V}^{-1} \mathrm{~s}^{-1}$ ). Another possible explanation for the lower $\sigma_{\mathrm{d}}$ of $p$-type patterns is their lower crystalline fraction, compared to their $n$-type counterparts, under the same patterning conditions. When writting on a $\mathrm{p}^{+}$-a-Si:H layer using the parameters that yielded the highest

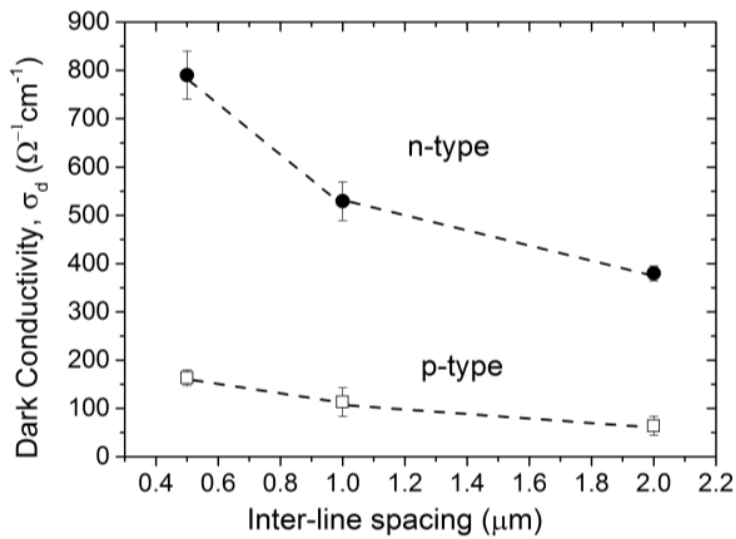

Figure 10 Dark conductivity, $\sigma_{\mathrm{d}}$, as a function of inter-line spacing, for line-spacing $0.5,1$ and $2 \mu \mathrm{m}$, measured on two samples $-n$ - and $p$-type, respectively - written with the same patterns that were used for C-AFM measurements 
$n$-type $\sigma_{\mathrm{d}}\left(\sigma_{\mathrm{d}}=886 \Omega^{-1} \mathrm{~cm}^{-1}, X_{\mathrm{C}}=98 \%\right)$, the $p$-type pattern has $X_{\mathrm{C}}=72 \%\left(\sigma_{\mathrm{d}}=165 \Omega^{-1} \mathrm{~cm}^{-1}\right)$.

It is also evident from Fig. 10 that, for both $n$ - and $p$-type films, as the inter-line distance becomes smaller, $\sigma_{\mathrm{d}}$ increases. This is a consequence of two effects: on the one hand, the crystalline fraction of the mesoscopic area increases as the inter-line spacing becomes smaller; on the other hand, Raman measurements show that the crystalline fraction of the crystallized lines slightly increases as they become closer together: $X_{\mathrm{C}}=81,83$ and $85 \%$ for inter-line spacing of 2, 1 and $0.5 \mu \mathrm{m}$, respectively. The Raman spectra of the (amorphous) inter-line regions, for 1 and $2 \mu \mathrm{m}$ spacing, show zero crystalline fraction, thus confirming the conductivity maps obtained by C-AFM. For $0.5 \mu \mathrm{m}$ spacing, the Raman analysis of the region between lines shows a small $X_{\mathrm{C}} \sim 30 \%$, but this is attributable to the fact that this distance is below the lateral micro-Raman resolution, and therefore these spectra include a contribution from adjacent crystallized areas.

\subsection{Piezoresistive properties of crystallized pat-} terns Doped nanocrystalline thin films are normally piezoresistive $[9,10]$, i.e. their conductivity changes upon application of strain. This is a very useful property which is used to build strain gauges and shape sensors of many types [11]. We therefore looked for the piezoresistive response of our structures, consisting of laser-crystallized doped Si patterns.

The piezoresistive effect is linear in strain, up to a certain strain level. Therefore it can be quantified by a characteristic gauge factor, $G F$, defined as $G F=\left(\Delta R / R_{0}\right) / \varepsilon$, where $R_{0}$ is the resistance of the un-deformed sample, $\Delta R=R-R_{0}$ is the change of resistance for a particular strain, and $\varepsilon$ is strain. In order to apply strain we performed 4-point bending experiments. Samples were fabricated on thin c-Si wafers $(250 \mu \mathrm{m}$ thick) coated with a $200 \mathrm{~nm} \mathrm{SiO} 2$ layer by CVD. The a-Si:H writing medium was a $150 \mathrm{~nm}$ thin film (both for $n$ - and $p$ type). The patterns consisted of $200 \mu \mathrm{m}$ long parallel lines with inter-line spacing of $1 \mu \mathrm{m}$, in a set of samples, and 0.5 $\mu \mathrm{m}$ inter-line spacing, in a second set, and were written in the centre of the substrates. The lines were irradiated at 40 $\mathrm{mW}$ laser power and $62 \mu \mathrm{m} / \mathrm{s}$ scan speed. The structures were contacted by metal ( $\mathrm{AlSiCu}$ ) parallel contacts defined by standard lithography, forming resistors $100 \mu \mathrm{m}$ long and $80 \mu \mathrm{m}$ wide. Samples were then cut in rectangular plates 35 $\mathrm{mm}$ long and $15 \mathrm{~mm}$ wide. The silicon plates were then introduced in the 4-point bending bridge (Fig.11a)). The 4point bending geometry is such that, upon bending, the free surfaces of the plate are forced to acquire a constant curvature between the two inner loading bars of the 4-point bridge. One of these surfaces becomes convex and tensile strain develops there, whereas the second surface is still parallel to the first and becomes concave, thus developing compressive strain. In our experiments, the patterned resistors were always placed on the tensile surface of the specimen, sitting between the two inner loading bars of the 4-point bridge (see Fig.11a)). Under such conditions, the in-plane tensile strain
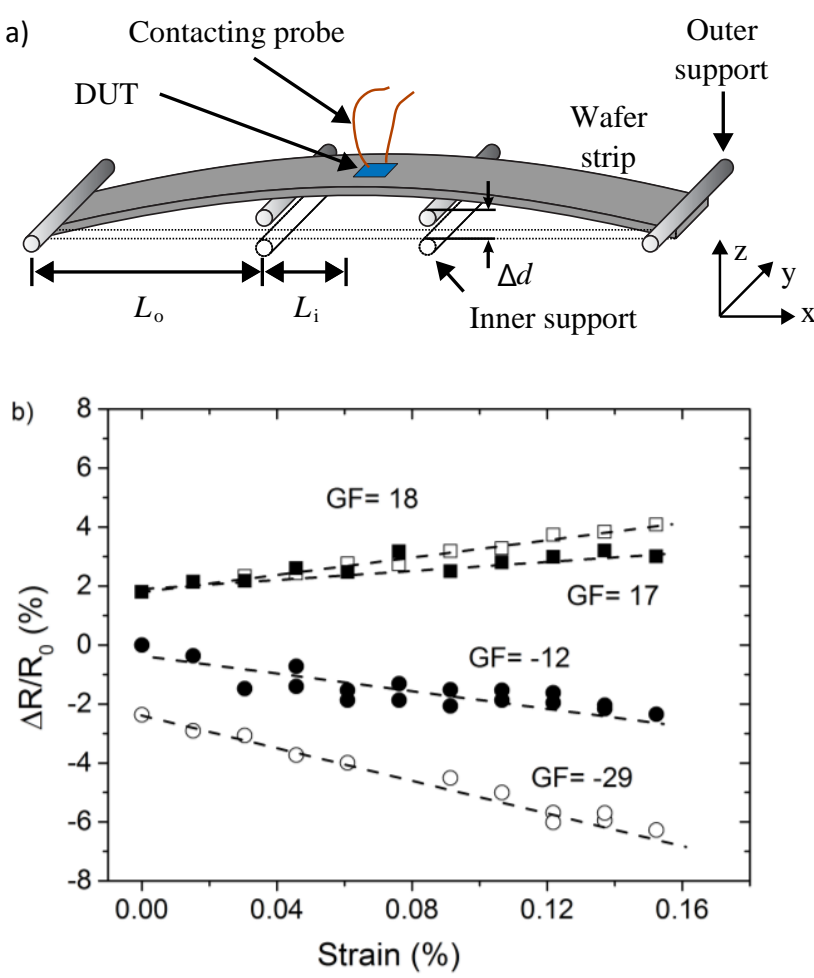

Figure 11 a) Schematics of the four-point bending bridge. Lo is the distance between the first and second loading bars. Lo $+\mathrm{Li}$ is one half the distance between the outward loading bars. b) Relative resistance change as a function of applied strain for $n$ (circles) and $p$-type (squares) nc-Si patterned resistors with $2 \mu \mathrm{m}$ (solid marks) and $0.5 \mu \mathrm{m}$ (open marks) line separation. The dashed lines are least squares adjustments to the data. The slope of the dashed lines is the gauge factor, GF.

developed is normal and uniaxial along the longitudinal axis of the sample and is given by:

$$
\varepsilon_{1}=\frac{6 t}{2 L_{0}^{2}+6 L_{0} L_{i}} \Delta d
$$

where $t, \Delta d, L_{0}$, and $L_{\mathrm{i}}$ denote the thickness of the sample, the displacement of the inner bars with respect to the outer bars, the distance between the inner and outer loading bars $\left(L_{0}=18 \mathrm{~mm}\right)$, and half the inner inter-bar distance $\left(L_{\mathrm{i}}=10\right.$ $\mathrm{mm})$. The resistance of the device under test (DUT) was then measured for a sequence of values of applied strain, calculated using eq.(2) for a corresponding sequence of displacements $\Delta d$. Applied strain was between 0 and $0.15 \%$. In these measurements the direction of current flow was always in a direction parallel to the uniaxial strain. Therefore the measured $G F$ s are the longitudinal ones (as opposed to transverse $G F$ s which were not addressed in the present work).

Fig.11b) shows the results of such measurements for $n$ (circles) and $p$-type (squares) nc-Si patterned resistors with $2 \mu \mathrm{m}$ (solid marks) and $0.5 \mu \mathrm{m}$ (open marks) line separation. The dashed lines are least squares adjustments to the data. 
The slope of the dashed lines is the gauge factor, GF. The first conclusion that stands out from the data is that the crystallized patterns have piezoresistive properties, with $G F>0$ for $p$-type resistors, and $G F<0$, for $n$-type resistors. The second conclusion is that patterns with inter-line spacing 0.5 $\mu \mathrm{m}$ have stronger piezoresistive response - translated in higher absolute value of $G F(G F=+18$ and -29$)$ - than patterns with inter-line spacing $1 \mu \mathrm{m}(G F=+17$ and -12$)$.

Regarding the first conclusion, it is observed that the patterned resistors have similar behaviour to directly deposited nc-Si:H films, as concerns the correlation between the sign of the $G F$ and the doping type $[10,12]$. As for the second conclusion, $G F$ s found in the present study fall in the same range of values as those encountered in our previous study of piezoresistance in nc-Si:H films, although they are lower than the highest values reported then [10]. The fact that patterns with inter-line separation of $0.5 \mu \mathrm{m}$ have higher $G F$ than those with $1 \mu \mathrm{m}$ separation correlates well with the results of the study by C-AFM presented in section 3.2, where the highest $\sigma_{\mathrm{d}}$ and $X_{\mathrm{C}}$ were obtained for $0.5 \mu \mathrm{m}$ line separation. It was shown there that, at this separation, adjacent lines essentially coalesce giving rise to a continuously crystallized surface. It was also shown in 3.2 that both $\sigma_{\mathrm{d}}$ and $X_{\mathrm{C}}$ were higher in $n$-type patterns than in $p$-type ones, which perfectly correlates with the obtained values of $G F$.

4 Conclusion A study was presented that shows that laser writing on doped a-Si:H thin films has the potential to become a valuable tool for patterning simple devices, like resistors, conductive lines and contacts, on hard and soft substrates, such as Si wafers or glass and plastic substrates. A systematic study was performed on each type of substrate to find the laser threshold power for crystallization, followed by writing at different laser fluences above the threshold power. The laser threshold power for crystallization of the films increased in the order PET and PI (4 mW), glass (7 $\mathrm{mW})$, and $\mathrm{SiO}_{2}(15 \mathrm{~mW})$ at a writing speed of $62 \mu \mathrm{m} / \mathrm{s}$. The highest conductivity for each substrate was $75 \Omega^{-1} \mathrm{~cm}^{-1}$, for PI, $390 \Omega^{-1} \mathrm{~cm}^{-1}$ for glass, and $886 \Omega^{-1} \mathrm{~cm}^{-1}$ for $\mathrm{Si} / \mathrm{SiO}_{2}$, obtained at a laser power of 4,20 and $40 \mathrm{~mW}$, respectively. It was shown that on hard substrates, namely on c-Si/SiO $\mathrm{S}_{2}$ wafers, it is possible to tune the electrical conductivity of the mesoscopic samples in a very broad range of values $\left(\sim 10^{-4}\right.$ $\left.-10^{3}\right)$ just by design of the pattern to be transferred. These patterns can be generated by a computer program that creates text files containing a set of instructions that can be read by the electronics that control the laser shutter, the distance of the objective lens above the sample, and the X-Y movement of the stage onto which the sample is mounted.

The piezoresistive response of patterned resistors on thin c-Si $/ \mathrm{SiO}_{2}$ wafers was demonstrated both for $n$ - and $p$-type films with gauge factors that depend on the pattern used. Maximum values of $G F$ were measured on patterns consisting of lines with $0.5 \mu \mathrm{m}$ line separation: $G F=+18$ and -29 for $p$ - and $n$-type patterns, respectively. These results are promising for the fabrication of strain gauges using the technology developed in this paper.
Acknowledgements The authors acknowledge Witec $\mathrm{GmbH}$ for collaboration in disclosing the set of instructions needed to communicate with Witec Four software.

\section{References}

[1] P. Fojtík, K. Dohnalová, T. Mates, J. Stuchlík, I. Gregor, J. Chval, A. Fejfar, J. Kočka and I. Pelant, Phil. Mag. B 82 (17), 1785 (2002).

[2] P. Roca i Cabarrocas, N. Layadi, T. Heitz, B. Drévillon, and I. Solomon, Appl. Phys. Lett. 65 (26), 3335 (1994).

[3] S.A. Filonovich, M. Ribeiro, A.G. Rolo, P. Alpuim, Thin Solid Films 516, 576 (2008).

[4] F. Gontad, J.C. Conde, S. Filonovich, M.F. Cerqueira, P. Alpuim, S. Chiussi, Thin Solid Films 536, 147 (2013).

[5] https://github.com/jborme/dpps.

[6] See, for example: M. Born, E. Wolf and A. Bathia, Principles of Optics (Cambridge University Press, Cambridge, 1999), chap. 8.

[7] O. Vetterl, F. Finger, R. Carius, P. Hapke, L. Houben, O. Kluth, A. Lambertz, A. Muck, B. Rech, H. Wagner, Sol. Energy Mater. Sol. Cells 62, 97 (2000).

[8] http://www.cleanroom.byu.edu/ResistivityCal.phtml.

[9] S. Nishida, M. Konagai, and K. Takahashi, Jpn. J. Appl. Phys. 25, 17 (1986).

[10] P. Alpuim, J. Gaspar, P. Gieschke, C. Ehling, J. Kistner, N. J. Gonçalves, M. I. Vasilevskiy, and O. Paul, J. Appl. Phys. 109, 123717 (2011).

[11] W. G. Pfann and R. N. Thurston, J. Appl.Phys. 32, 2008 (1961).

[12] D. Lange, P. Roca i Cabarrocas, N. Triantafyllidis \& D.Daineka, Sol. Energy Mater. Sol. Cells (2015) doi:10.1016/j.solmat.2015.09.014 